\title{
ON PROPERTIES OF REGRESSIVE SETS
}

\author{
BY
}

K. I. APPEL AND T. G. MCLAUGHLIN

1. Introduction. It was recently demonstrated by $R$. Mansfield (unpublished) that complementary retraceable sets must be recursive. Our main result, proved in $\$ 3$, is that at least one member of any complementary pair of regresssive sets is recursively enumerable. This is a generalization of Mansfield's theorem, but the method of proof, in $\$ 3$, is quite different. In $\$ 4$, one of the two principal lemmas used by Mansfield is generalized, and some related material is developed, including an alternative derivation of the main theorem. In $\$ 5$, we show that if the intersection of a pair of regressive sets is infinite then it has an infinite regressive subset. As a corollary to this last result, we prove that a coregressive hypersimple set is many-one incomparable with any hyperhypersimple set.

2. Definitions and notations. An infinite set $\alpha$ of numbers is regressive (see [1]) iff there exists a partial recursive function $g$, and a nonrepetitive listing $b_{0}, b_{1}, \cdots$ of the elements of $\alpha$, such that

(1) $\alpha \subset \delta g$, and

(2) $g\left(b_{0}\right)=b_{0}, \quad(\forall n)\left(g\left(b_{n+1}\right)=b_{n}\right)$.

(The notation ' $\delta$ ', as well as ' $\rho$ ', '+', ' $'$ ', '*', and ' $\cong$ ', is used as in [1].) An infinite set $\alpha$ of numbers is said to be retraceable iff $\alpha$ is regressive, in the above sense, with $b_{0}, b_{1}, \ldots$ specified to be the listing of $\alpha$ in order of magnitude. If $\alpha$ is regressive (retraceable) with respect to the listing $b_{0}$, $b_{1}, \cdots$ of its elements and the partial recursive function $g$, we say that $g$ regresses (retraces) $\alpha$, or is a regressing (retracing) function for $\alpha$, and that $b_{0}$ is the root of $\alpha$ under $g$.

We shall freely use the results of [1] and [4]. In particular, we make use of the fact that if $g$ regresses $\alpha$ with respect to the listing $b_{0}, b_{1}, \ldots$ of $\alpha$, then there is a partial recursive subfunction $g_{0}$ of $g$ which also regresses $\alpha$ with respect to the listing $b_{0}, b_{1}, \ldots$ and is "special" in the sense that

(a) $\rho g_{0} \subset \delta g_{0}$, and

(b) $x \in \delta g_{0} \Longrightarrow(\exists n)\left(g_{0}^{n}(x)=b_{0}\right)$.

With the exception of our discussion of Theorem 3 in $\$ 4$, whenever we make use of regressing functions they will be special in the above sense.

Given a partial number-theoretic function $g$, we set

$$
\hat{g}(x)=d f\left\{y \mid(\exists n)\left(g^{n}(x)=y\right)\right\} .
$$

Received by the editors December 19, 1963. 
$\left(\hat{g}(x)=\{x\}\right.$ if $x \notin \delta g$, by our adherence to the usual convention that $g^{0}(x)$ $=x$.)

By the "standard computation" of two partial recursive functions $g$ and $h$ for which definite computation procedures are given, we mean the algorithm (relative to the given fixed procedures for computing $g$ and $h$ ) which proceeds as follows:

Stage 1. Proceed one step in the computation to find $g(0)$; then go to Stage 2.

Stage 2. Proceed one step in the computation to find $h(0)$; then go to Stage 3.

Stage $2 n+1(n>0)$. Proceed one more step in the computation to find $g(r)$, where $r+1$ is the number of distinct prime factors of $2 n+1$, provided $g(r)$ has not previously been found; then go to Stage $2 n+2$.

Stage $2 n+2(n>0)$. Same as Stage $2 n+1(n>0)$ with $g$ replaced by $h, 2 n+1$ replaced by $2 n+2$, and $2 n+2$ replaced by $2 n+3$.

In $\$ \$ 3$ and 5, we use the symbol ' $C$ ' to refer to the standard computation of two given partial recursive functions $g$ and $h$ (assuming fixed computation procedures for $g, h$ ).

Following recent convention, we denote by ' $J(\alpha, \beta)$ ' the standard l.u.b., $\{2 n \mid n \in \alpha\}+\{2 n+1 \mid n \in \beta\}$, of the number sets $\alpha$ and $\beta$.

\section{The main result.}

Theorem 1. If $\alpha, \alpha^{\prime}$ are both regressive, then at least one of them is r.e.

We assume, in order to prove Theorem 1 by reductio ad absurdum, that there exist complementary immune sets $\alpha$ and $\beta$, both regressive. Let $g$ and $h$ be partial recursive functions which (specially) regress $\alpha$ and $\beta$, respectively. In what follows, a definition, remark, or lemma is preceded by the symbol ' $\dagger$ ' if it is to be read both as it stands and with $g, \alpha$ interchanged, at each occurrence, with $h, \beta$, respectively.

$\dagger$ Definition. $D_{g}^{\beta}=d f\{x \in \beta \mid x \in \delta g\}$.

Let $\left\{x_{i}\right\}$ be a nonrepetitive listing of $D_{g}^{\beta},\left\{y_{i}\right\}$ a nonrepetitive listing of $D_{h}^{\alpha}$.

†REMARK A. $\delta g$ is r.e.; hence, since $\alpha$ is immune, $D_{g}^{\beta}$ is infinite.

†REMARK B. $(\forall a \in \beta)(\forall b \in \alpha)(a \notin \hat{g}(b))$.

†Remark C. $(\forall z)(\forall w)(z \notin \hat{g}(w) \& w \notin \hat{g}(z) \Longrightarrow z \in \beta \bigvee w \in \beta)$.

(Remark $\mathrm{C}$ follows simply from the definition of regressing function.)

†REMark D. $(\forall z)(\forall w)([(z \in \beta \bigvee w \in \beta) \& z \in h(w)] \Longrightarrow z \in \beta)$.

$\dagger$ Lemma 1.1. There are only finitely many $x_{j} \in D_{g}^{\beta}$ such that $\left(\exists y_{i} \in D_{h}^{a}\right)$ $\left(y_{i} \notin \hat{g}\left(x_{j}\right) \& x_{j} \in \hat{h}\left(y_{i}\right)\right)$.

Proof of Lemma 1.1. Suppose infinitely many $x_{j}$ 's satisfy the condition of the lemma. We construct, on this hypothesis, an enumeration of an 
infinite r.e. subset $\tau$ of $\beta$; since $\beta$ is immune, Lemma 1.1 will follow. Begin $\mathrm{C}$ for $g$ and $h$ (see $\$ 2$ ). As soon as enough information is developed by $\mathrm{C}$ to show that for a pair $w, z$ of numbers we have

(1) $g(w), h(w), g(z), h(z)$ all exist, and

(2) $z \notin \hat{g}(w), w \notin \hat{g}(z), z \in \hat{h}(w)$, and $w \notin \hat{h}(z)$, we place $z$ in our generated set $\tau$. Now, in particular, every pair $\left(x_{j}, y_{i}\right)$ of the infinitely many pairs of our reductio ad absurdum hypothesis satisfies (1) and (2), with $x_{j}$ for $z$ and $y_{i}$ for $w$; thus $\tau$ is infinite. By Remarks $\mathrm{C}$ and $\mathrm{D}, \tau \subset \beta$. ।

$\dagger$ Lемма 1.2. There are only finitely many $x_{j} \in D_{g}^{\beta}$ such that $\left(\exists y_{i} \in D_{h}^{\alpha}\right)$ $\left(y_{i} \in \hat{g}\left(x_{j}\right) \& x_{j} \in h\left(y_{i}\right)\right)$.

Proof of Lemma 1.2. Assume the contrary. Let $T=\left\{\left(x_{j}, y_{i}\right) \mid y_{i} \in \hat{g}\left(x_{j}\right)\right.$ $\left.\& x_{j} \in \hat{h}\left(y_{i}\right)\right\}$; our assumption entails that $T$ is infinite. We proceed in a way analogous to our argument proving Lemma 1.1; but now we consider quadruples of (pairwise distinct) numbers $w, z, s, t$ such that

(1) $g$ and $h$ are both defined on each of $w, z, s, t$;

(2) $z \notin \hat{g}(t)$ and $t \notin \hat{g}(z)$;

(3) $z \in \hat{h}(s)$ and $s \in \hat{h}(\dot{t})$.

We place $z$ in our generated set $\tau$ when, and only when, $\mathrm{C}$ has provided the information that (1)-(3) hold for a quadruple $(w, x, s, t)$ of distinct numbers. First, we note that the set $\tau$ thus generated is a subset of $\beta$. For by (2), (3), and Remarks C and D, $z$ must be in $\beta$. Next, we verify that $\tau$ is infinite, which presents a contradiction to the immunity of $\beta$. Let $\left(x_{j}, y_{i}\right)$ $\in T$ : we claim that there is a second pair $\left(x_{k}, y_{l}\right) \in T$ satisfying

(4) $x_{j} \in \hat{h}\left(x_{k}\right)-\left\{x_{k}\right\}, y_{i} \in \hat{g}\left(y_{l}\right)-\left\{y_{l}\right\}$.

Assume the contrary. Let $Y=g\left(y_{i}\right), X=h\left(x_{j}\right)$. For any pair $\left(x_{k}, y_{l}\right)$, if $\left(x_{k}, y_{l}\right)$ does not satisfy (4) then $x_{k} \in X$ or $y_{l} \in Y$. Therefore, since $T$ is infinite, one of these alternatives, say $x_{k} \in X$, holds for infinitely many $\left(x_{k}, y_{l}\right) \in T$. By hypothesis, $\left(x_{k}, y_{l}\right) \Longrightarrow y_{l} \in g\left(x_{k}\right)$. Hence, there is a set $S \subset T$ such that $S$ is infinite and $\left(x_{k}, y_{l}\right) \in S \Longrightarrow\left(x_{k}, y_{l}\right) \in X \times \bigcup_{z \in X} \hat{g}(z)$. But this latter set is finite, and we have a contradiction. (Clearly, if the other alternative is selected a similar contradiction arises.)

Now, if $\left(x_{j}, y_{i}\right) \in T$, we see that there exists a pair $\left(x_{k}, y_{l}\right) \in T$ such that (1)-(3) hold with $x_{j}$ for $z, y_{i}$ for $w, x_{k}$ for $s$, and $y_{l}$ for $t$ : this follows from what was just proved above (concerning (4)) and the facts that $g\left(x_{j}\right)$ is finite and $\left(z_{1} \in \hat{h}\left(z_{2}\right) \& z_{2} \in \hat{h}\left(z_{3}\right)\right) \Longrightarrow z_{1} \in \hat{h}\left(z_{3}\right)$. Thus, $\tau$ is infinite. I

†LEMMA 1.3. $\beta$ is recursively enumerable.

Proof of Lemma 1.3. By Lemmas 1.1 and 1.2, there exists a number $m$ such that

$$
\left(x_{j} \in D_{g}^{\beta} \&\left(\exists y_{i} \in D_{h}^{\alpha}\right)\left(x_{j} \in \hat{h}\left(y_{i}\right)\right)\right) \Longrightarrow x_{j}<m .
$$


We enumerate an infinite subset $\tau$ of $\beta$ as follows: let $b_{0} \in D_{g}^{\beta}$ be such that $b_{0}>m$. Begin $C$. When sufficient information has unfolded to show that for a number $z$ we have $z>b_{0}, h$ and $g$ are both defined on $z$, and $b_{0} \in h(z)$, we put $z$ into $\tau$. If such a $z$ were an element of $D_{h}^{\alpha}$, we would have, from $b_{0} \in h(z)$, a contradiction to the choice of $b_{0}$; hence, any such $z$ is in $D_{g}^{\beta} \subset \beta$. Since $D_{g}^{\beta}$ is infinite, $\tau$ is infinite. Since $\beta$ is regressive and has an infinite r.e. subset, $\beta$ is r.e. I

Since Lemma $1 . \overline{3}$ contradicts our hypothesis that $\beta$ is immune, Theorem 1 follows.

CoRollary 1.4 (MANSFIELd). Complementary retraceable sets are recursive.

We observe that Yates, in [4], indicated a way of showing the existence of hyperhyperimmune retraceable sets.

COROLLARY 1.5. A hyperhyperimmune regressive set must have a nonregressive complement.

Proof. Let $\alpha$ be hyperhyperimmune and regressive. If $\alpha^{\prime}$ were regressive, then, by Theorem 1, $\alpha^{\prime}$ would be r.e. and hence hyperhypersimple. But r.e. sets with regressive complement are not hyperhypersimple, and Corollary 1.5 follows. I

In $\$ 4$, we strengthen Corollary 1.5 by showing that, moreover, the complement of an immune regressive set must fall far short of being hyperimmune.

REMark. Reflection on the proof of Theorem 1 leads at once to the observation that if $\alpha$ is a coregressive hypersimple set (i.e., if $\alpha$ is hypersimple and $\alpha^{\prime}$ is regressive) and $p$ is a partial recursive function which regresses $\alpha$, then $\delta p-\alpha$ must be finite. In $\$ 4$, we will prove (Theorem 3 ) a substantially more inclusive assertion.

4. Regressive sets which are immune but not hyperimmune. To prove that complementary retraceable sets are recursive, Mansfield made use of the following two lemmas $\left({ }^{1}\right)$ :

LEMMA (i). If $\alpha$ is a retraceable set having exactly one element in common with each pair $(2 k, 2 k+1)$, then $\alpha$ is recursive.

LEMMA (ii). If $\alpha, \alpha^{\prime}$ are retraceable, so is $J\left(\alpha, \alpha^{\prime}\right)$.

Lemma (i) admits a generalization to the regressive case which we state and prove below as Theorem 2. The failure of the regressive analogue of Lemma (ii) is documented in Corollary 2.1 below.

If $\alpha$ is any set of numbers, we denote by ' $|\alpha|$ ' the cardinal of $\alpha$. In

( ${ }^{1}$ We are indebted to Professor Dekker for our knowledge of Mansfield's approach to the retraceable case. 
place of the notation ' $\rho_{k}$ ' of [1], we use the notation ' $D_{k}$ '.

DEFintion. A sequence $\left\{D_{\varphi(n)}\right\}$ of number sets, $\varphi$ a recursive function, is called a discrete array with rows of bounded cardinality iff (i) $\left\{D_{\varphi(n)}\right\}$ is a discrete array in the usual sense (i.e., in the language of $[4],\left\{D_{\varphi(n)}\right\}$ is a "strongly r.e. class of pairwise-disjoint, nonempty finite sets"), and (ii) $(\exists k \geqq 1)(\forall n)\left(\left|D_{\varphi(n)}\right| \leqq k\right)$.

Theorem 2. Let $\left\{D_{\varphi(n)}\right\}$ be a discrete array with rows of bounded cardinality and $k$ the maximal cardinal of any $D_{\varphi(n)}$. Let $\alpha$ be a regressive set such that $D_{\varphi(n)} \cdot \alpha \neq \emptyset$ holds for all $n$. Then $\alpha$ is r.e.

Proof. Let $p$ be a partial recursive function which regresses $\alpha$ relative to the listing $a_{0}, a_{1}, \ldots$ of the elements of $\alpha$. We shall call a number $n$ a $(p, j, b)$-knot $(1 \leqq j \leqq k)$ iff there exist $j$ elements $u_{q}$ of some set $D_{\varphi(m)}$ for which $n \in \hat{p}\left(u_{q}\right), 1 \leqq q \leqq j$, and $b \in \hat{p}(n)$. Now, let $j$ be the largest number such that $\left\{a_{i} \mid a_{i} \in \alpha \& a_{i}\right.$ is a $\left(p, j, a_{0}\right)$-knot $\}$ is infinite. Clearly $1 \leqq j \leqq k$. Let $b$ be an element of $\alpha$ such that for all $a_{i} \in \alpha$, if $a_{i}$ is a $\left(p, h, a_{0}\right)$-knot for $j<h \leqq k$, then $a_{i} \in \hat{p}(b)-\{b\}$. Next let $\left\{D_{\psi(m)}\right\}$ be the subarray of $\left\{D_{\varphi(m)}\right\}$ consisting of exactly those rows $D_{\varphi(m)}$ such that $a_{i} \in \alpha \& a_{i} \in D_{\varphi(m)}$ $\Longrightarrow b \in \hat{p}\left(a_{i}\right)-\left\{a_{i}\right\}$. If $a$ is a $(p, j, b)$-knot obtained from $\left\{D_{\downarrow(m)}\right\}$ then $a \in \alpha$, for otherwise $b$ would be a $\left(p, h, a_{0}\right)$-knot for some $h>j$. By definition of discrete array and regressing function, the set $\gamma$ of all $(p, j, b)$ knots obtained from $\left\{D_{\psi(m)}\right\}$ is r.e. and by definition of $j, \gamma$ is infinite. Thus, $\alpha$ has an infinite r.e. subset, hence $\alpha$ is itself r.e. $\left({ }^{2}\right.$ ). (As a matter of fact, $\gamma$ is all but finitely much of $\alpha$.) I

Notice that Mansfield's Lemma (i) is a corollary to Theorem 2, since any r.e. set having exactly one element in common with each pair $(2 k, 2 k+1)$ must, clearly, be recursive. We observe that, in contrast to the retraceable case, it is not always true that if $\alpha, \alpha^{\prime}$ are regressive, then $J\left(\alpha, \alpha^{\prime}\right)$ is also regressive. In fact, we have

Corollary 2.1. $J\left(\alpha, \alpha^{\prime}\right)$ is regressive $\Longleftrightarrow \alpha$ is recursive.

Proof. If $\alpha$ is recursive, then so, clearly, is $J\left(\alpha, \alpha^{\prime}\right)$; hence, the latter set is regressive. On the other hand, suppose $J\left(\alpha, \alpha^{\prime}\right)$ is regressive. Then, in view of Theorem 2, it is r.e.; therefore, by reason of sharing exactly one element with each pair $(2 k, 2 k+1)$, it is recursive. But then $\alpha=$ $\left\{x \mid 2 x \in J\left(\alpha, \alpha^{\prime}\right)\right\}$ is recursive. I

In particular, if $\beta$ is a hypersimple set with regressive complement, then,

$\left(^{2}\right)$ It might be remarked that by a proof similar to the proof of Theorem 2 we can establish the following characterization of recursively infinite number sets.

THEOREM. $\alpha$ is recursively infinite $\Longleftrightarrow$ there exists an r.e. binary relation $R$, and discrete array $\left\{D_{\varphi(n)}\right\}$ with rous of bounded cardinality such that: (i) $\alpha \cdot D_{\varphi(n)} \neq \emptyset$ holds for all $n$; (ii) $(x \in$ $\alpha \cdot \bigcup_{n} D_{\varphi(n)} \& y \in \bigcup_{n} D_{\varphi(n)}$ \& there is an R-chain from $x$ to $\left.y\right) \Longrightarrow y \in \alpha$ (iii) $y \in \alpha \cdot \bigcup_{n} D_{\varphi(n)} \Longrightarrow$ $(\exists m)\left(x>m \& x \in \alpha \cdot \bigcup_{n} D_{\varphi(n)} \Longrightarrow\right.$ there is an R-chain from $x$ to $\left.y\right)$. 
although both $\beta$ and $\beta^{\prime}$ are regressive, $J\left(\beta, \beta^{\prime}\right)$ is not regressive. (This last assertion is as a matter of fact easy to prove directly, i.e., without going through Theorem 2.)

CoRollary 2.2. If $\alpha$ is regressive and immune, but not hyperimmune, and if $\left\{D_{\varphi(n)}\right\}$ is any discrete array witnessing the nonhyperimmunity of $\alpha$, then, for every $k \geqq 1$, only finitely many $D_{\varphi(n)}$ 's have power $\leqq k$.

Proof. By Theorem 2 and the fact that one can effectively list the subsequence of $D_{\varphi(n)}$ 's such that $\left|D_{\varphi(n)}\right| \leqq k$, noting that whenever that subsequence has infinitely many terms we have a subarray.

Corollary 2.2 is similar to the following proposition, which is implicit in Post [3] but seems to have escaped explicit notice in the literature.

Proposition 2.3. Let $\zeta$ be a simple set which is not hypersimple, let $k$ be a positive integer, and let $\left\{D_{\varphi(n)}\right\}$ be any discrete array witnessing the nonhypersimplicity of $\zeta$. Then only finitely many rows of $\left\{D_{\varphi(n)}\right\}$ have cardinality $\leqq k$.

Proof. We could base the proof on Post's result [3] that no simple set is bounded-truth-table complete; instead, we give a simple direct argument. Suppose there is a positive integer $k$ such that $\left|D_{\varphi(n)}\right|=k$ for infinitely many $n$; let $k_{0}$ be the least such $k$. Let $\left\{D_{\psi(n)}\right\}$ be the subarray of $\left\{D_{\varphi(n)}\right\}$ consisting of the rows with cardinality $k_{0}$. Now, there is a maximal $j$, $1 \leqq j<k_{0}$, such that infinitely many rows of $\left\{D_{\psi(n)}\right\}$ contain $j$ members of $\zeta$; let $j_{0}$ be this maximal $j$. Then, clearly, we obtain an infinite r.e. subset of $\zeta^{\prime}$ by finding those $D_{\psi(n)}$, for sufficiently large $n$, which contain $j_{0}$ elements of $\zeta$ and listing the other $k_{0}-j_{0}$ members of $D_{\psi(n)}$. Proposition 2.3 follows.

Remarks. (1) It follows from Proposition 2.3 that if we add to the hypotheses of Theorem 2 the assumption that $(\forall n)\left(D_{\psi(n)} \nsubseteq \alpha\right)$ where $\left\{D_{\psi(n)}\right\}$ is some subarray of $\left\{D_{\varphi(n)}\right\}$, then the conclusion becomes: $\alpha$ is a nonsimple r.e. set.

(2) Bearing Proposition 2.3 in mind, it is easy to see that if $\zeta$ is a simple set and $\left\{\omega_{\varphi(n)}\right\}$ is an r.e. sequence of pairwise-disjoint, nonempty finite sets witnessing that $\zeta$ is not hyperhypersimple, then $(\forall k \geqq 1)(\exists n)\left(\left|\omega_{\varphi(n)}\right|>k\right)$. On the other hand, it can be shown that if $\zeta$ is simple, there exists a simple set $\zeta_{0}$ such that (a) $\zeta_{0} \equiv_{m} \zeta$, and (b) for some r.e. sequence $\left\{\omega_{\varphi(n)}\right\}$ of pairwisedisjoint nonempty finite sets witnessing nonhyperhypersimplicity of $\zeta_{0}$, we have $(\forall n \geqq 1)\left(\mid\left\{k: \omega_{\varphi(k)}\right.\right.$ is an $n$-element subset of $\left.\left.\zeta_{0}^{\prime}\right\} \mid=\boldsymbol{\aleph}_{0}\right)$.

Given Proposition 2.3, we are in a position to prove, independently of Theorem 1, a result somewhat stronger than the remark at the end of $\$ 3$.

THeOREM 3. If $\alpha$ is simple and $p$ is a partial recursive function which regresses $\alpha$ (not assuming condition (b) of $\$ 2$ for $p$, but rather condition (4) of $[1, p .81])$, then $\delta p-\alpha$ is finite. 
Proof. Suppose $\delta p-\alpha$ were infinite. Let $s(p, n)=\left\{y \mid p^{*}(y)=n\right\}$. We note that $s(p, n)$ is r.e. and contains precisely one element of $\alpha$. Hence if $s(p, n)$ were infinite we would have an infinite r.e. subset of $\alpha^{\prime}$, contradicting the immunity of $\alpha^{\prime}$. Thus, for each $n, s(p, n)$ is finite. Therefore, by our initial assumption, we have $|s(p, n)| \geqq 2$ for infinitely many numbers $n$; and these numbers form an r.e. set. Let $\varphi$ be a 1-1 recursive function generating this set. Let $\psi$ be a recursive function such that, for each $n$, $D_{\psi(n)}$ consists of precisely two members of $s(p, \varphi(n))$. Then $\left\{D_{\psi(n)}\right\}$ is a discrete array, with rows of bounded cardinality, witnessing the nonhypersimplicity of $\alpha$. This state of affairs, however, contradicts Proposition 2.3, and Theorem 3 follows.

Corollary 3.1. Let $\alpha$ be simple, $p$ a partial recursive function which regresses $\alpha$ (in the sense of $[1, p .81])$. Then $p$ is not potentially recursive.

Proof. Suppose, to the contrary, that $f$ is a recursive extension of $p$. First note that $\hat{f}(x)$ is finite for all $x$. For if there is a $k$ such that $f^{k}(x)=a$ $\in \alpha$, and $p^{*}(a)=l$, then $|\hat{f}(x)| \leqq k+l+1$. And if $\hat{f}(x) \subset \alpha^{\prime}$, then, since $\alpha$ is simple and $\hat{f}(x)$ is r.e., $f(x)$ must be finite. We now split the natural numbers into two sets $U$ and $V$ as follows:

$$
U=\left\{x \mid(\exists n)\left(f^{n+1}(x)=f^{n}(x)\right)\right\}, V=U^{\prime} .
$$

$U$ and $V$ are recursively enumerable, $V$ since it consists of just those $x$ for which $f(x)$ contains a cycle of two or more elements. Clearly, $V \subset \alpha^{\prime}$; hence $V$ is finite. Therefore, we see that $\left.f\right|_{U}$ is a partial recursive function, with cofinite domain, which regresses $\alpha$ in the sense of $[1$, p. 81]; since this contradicts Theorem 3, Corollary 3.1 is proved.

(We might note that condition (a) of $\$ 2$, for $p$, need not be assumed in Corollary 3.1. With regard to Theorem 3 itself: it can be seen that if $\alpha$ is maximal, then we need not assume $\rho p \subset \delta p$; whereas, there exist quasimaximal sets of rank two for which that assumption is indeed vital to Theorem 3.)

Remarks. (1) Corollary 3.1 holds true of pseudo-simple r.e. sets, as well as of simple ones.

(2) As one of the authors has elsewhere pointed out, there exist retraceable sets $\alpha$ none of whose retracing functions is potentially recursive; such an $\alpha$ must, of course, be immune. As a matter of fact, the following can be proved: there exists a retraceable set $\alpha$ such that

(i) $\beta$ recursive $\Longrightarrow \beta \cdot \alpha$ or $\beta^{\prime} \cdot \alpha$ is finite, and

(ii) $\alpha^{\prime}$ has no simple subset.

If we could prove the existence of an immune regressive set $\alpha$ satisfying (i) but not (ii), it would follow readily that no regressing function of $\alpha$ could be potentially recursive, and the immune-set analogue of Corollary 3.1 would be established. 
We point out, finally, an alternative way of proving Theorem 1 , based on Theorem 2. First, by examining the proof of Theorem 3, we see that the following proposition is true.

Proposition 3.2. Suppose $\alpha$ is a regressive set and $\alpha, \alpha^{\prime}$ are immune. Then $\alpha^{\prime}$ fails to be hyperimmune in that there is a discrete array $\left\{D_{\phi(n)}\right\}$ with rows of bounded cardinality (in fact, all having cardinality 2) such that $(\forall n)\left(D_{\varphi(n)} \cdot \alpha^{\prime} \neq \emptyset\right)$.

Now, let $\alpha$ be regressive; and suppose that $\alpha^{\prime}$ is likewise regressive, and that $\alpha, \alpha^{\prime}$ are both immune. By Proposition 3.2, $\alpha^{\prime}$ is penetrated by each row of a discrete array whose rows are of bounded cardinality; therefore, by Theorem $2, \alpha^{\prime}$ is r.e. This, however, contradicts the immunity of $\alpha^{\prime}$, and Theorem 1 follows.

(It can be shown, using a construction based on a suggestion by $P$. $R$. Young, that there do exist regressive sets which are immune and have immune complement.)

\section{The intersection of a pair of regressive sets.}

THEOREM 4. The intersection of two regressive sets, if infinite, has an infinite regressive subset.

Proof. Let $\alpha, \beta$ be regressive infinite number sets, regressed by $g$ and $h$, respectively; and suppose that $\alpha \cdot \beta$ is infinite. We can assume, without loss of generality, that the root, $b_{0}$, of $\alpha$ under $g$ is the same as the root of $\beta$ under $h$.

If $x$ and $y$ are distinct numbers such that $y \in \hat{g}(x), y \in h(x)$, and

$$
(\forall z)(z \neq x \& z \neq y \Longrightarrow y \notin h(z) \bigvee y \notin g(z) \bigvee z \notin h(x) \quad z \notin g(x)),
$$

then we refer to the pair $(x, y)$ as a loop. $x$ is called the upper vertex of the loop, and $y$ the lower vertex. We say that $x$ is rooted at the ith stage of $\mathrm{C}$ iff $x \in \delta g \cdot \delta h$ and either $x=b_{0}$ or, at some stage $k \leqq i$ of $\mathrm{C}$, enough computations have been made to show that

$$
\left(\exists y_{1}, \cdots, y_{k}\right)\left(\left(x, y_{1}\right),\left(y_{1}, y_{2}\right), \cdots,\left(y_{k-1}, y_{k}\right),\left(y_{k}, b_{0}\right) \in g\right) \bigvee\left(x, b_{0}\right) \in g
$$

and

$$
\left(\exists z_{1}, \cdots, z_{l}\right)\left(\left(x, z_{1}\right),\left(z_{1}, z_{2}\right), \cdots,\left(z_{l-1}, z_{l}\right)\left(z_{l}, b_{0}\right) \in h\right) \bigvee\left(x, b_{0}\right) \in h .
$$

A loop $(x, y)$ such that $x$ is rooted at the $i$ th stage of $\mathrm{C}$ is said to be acceptable at the ith stage of $\mathrm{C}$. We now describe a certain algorithm, $\mathfrak{A}$, for generating the pairs of a partial recursive function $f$ together with the domain, $F$, of $f$.

Step 0 of $\mathscr{U}$ : Put $b_{0}$ into $F$; then put $\left(b_{0}, b_{0}\right)$ into $f$; then go to Step 1 of $\mathscr{U}$.

Step $k$ of $\mathscr{A}, k>0$ : Perform the $k$ th stage of $C$. If no loops $(x, y)$ are then in evidence such that $(x, y)$ is acceptable at the $k$ th stage, $y \in F$, and $x$ $\notin F$ (i.e., $x$ is not yet in $F$ ), go on to Step $k+1$ of $\mathscr{U}$. If any such loops 
are in evidence, let

$$
\left(x_{1}, y_{11}\right), \cdots,\left(x_{1}, y_{1 k_{1}}\right),\left(x_{2}, y_{21}\right), \cdots,\left(x_{2}, y_{2 k_{2}}\right), \cdots,\left(x_{l}, y_{l_{1}}\right), \cdots,\left(x_{l}, y_{l_{l}}\right),
$$

with $x_{1}, \cdots, x_{l}$ mutually distinct, be the totality of them. For each $i, 1$ $\leqq i \leqq l$, choose the loop $\left(x_{1}, y_{i r}\right), 1 \leqq r \leqq k_{i}$, for which $y_{i r}$ is smallest; put $x_{i}$ into $F$, and then put $\left(x_{i}, y_{i r}\right)$ into $f$. In general, this procedure introduces further acceptable loops satisfying the extra conditions concerning $F$, so we must now repeat the procedure until no more such loops arise. (Clearly, since only finitely many stages of $C$ have been performed so far, we will run out of such loops after finitely many iterations of the indicated procedure.) When no more such loops appear, proceed to Step $k+1$ of $\mathfrak{A}$.

Lemma 4.1. $f$ is a partial recursive function.

Proof of Lemma 4.1. Clearly, $f$ is generated by an effective procedure (namely, $\mathscr{A})$; thus $f$ is an r.e. set of pairs. Now, whenever we put a pair $(x, y)$ into $f$, we must have just put its upper vertex, $x$, into $F$; thus, according to $\mathfrak{A}, x$ never subsequently appears as first member of a pair placed in $f$. Therefore, $f$ is a function, and so $f$ is a partial recursive function, and the proof of Lemma 4.1 is complete.

Lemma 4.2. Let $x \in \alpha \cdot \beta$, and let $s(x)$ be the least $i$ such that $x$ is rooted at the ith stage of $\mathrm{C}$. Then $x$ is placed in $F$ at Step $s(x)$ of $\mathfrak{A}$.

Proof of 4.2. Suppose otherwise: Let $x_{0}$ be a member of $\alpha \cdot \beta-\left\{b_{0}\right\}$ such that $x_{0}$ is not placed in $F$ at Step $s\left(x_{0}\right)$ of $\mathfrak{A}$, and such that $(\forall y \in \alpha \cdot \beta)$ $\left(y \in g\left(x_{0}\right) \& y \neq x_{0} \Longrightarrow y\right.$ is placed in $F$ at Step $s(y)$ of $\left.\mathscr{Q}\right)$. (Clearly $s\left(b_{0}\right)$ $=0$; and $b_{0}$ is placed in $F$ at Step 0 of $\mathscr{U}$. Also, note that $x_{0}$ certainly cannot be placed in $F$ at any Step $t$ of $\mathfrak{A}$ for which $t<s\left(x_{0}\right)$.) Suppose $y_{0}$ $\in \alpha \cdot \beta$ and $\left(x_{0}, y_{0}\right)$ is an acceptable loop at Step $s\left(x_{0}\right)$ of $\mathscr{U}$. (Such a $y_{0}$ must exist, since $x_{0}$ is rooted at Step $s\left(x_{0}\right)$ of $\mathscr{A}$.) Since $y_{0} \in \hat{g}\left(x_{0}\right) \cdot \hat{h}\left(x_{0}\right)$, we have $s\left(y_{0}\right) \leqq s\left(x_{0}\right)$; thus $y_{0}$ is put in $F$ at or before Step $s\left(x_{0}\right)$ of $\mathfrak{A}$. Therefore, at Step $s\left(x_{0}\right)$ of $\mathscr{U}$, we find $\mathfrak{A}$ ordering us to place $x_{0}$ in $F$ and some pair of the form $\left(x_{0}, w\right)$ into $f$, contradicting our assumption. Lemma 4.2 follows.

Lemma 4.3. For every $x \in \alpha \cdot \beta, x=b_{0} \bigvee\left(x, b_{0}\right) \in f \bigvee\left(\exists y_{1}, \cdots, y_{k} \in \alpha \cdot \beta\right)$ $\left(\left(x, y_{1}\right),\left(y_{1}, y_{2}\right), \cdots,\left(y_{k-1}, y_{k}\right),\left(y_{k}, b_{0}\right) \in f\right)$.

Proof of Lemma 4.3. By Lemma 4.2, $x \in F$. By the definition of $f$, if $(x, y) \in f$ then $y \in \alpha \cdot \beta-\{x\}$; and, in particular, $y \in \hat{g}(x) \cdot h(x)$. But $g(x), h(x)$ are finite, and so the lemma follows by "finite descent".

We may now complete the proof of Theorem 4. Set $c_{0}=b_{0}$; then $\left(c_{0}, c_{0}\right)$ $\in f$. Let $B_{1}$ be the set $\left\{x \in \alpha \cdot \beta \mid\left(x, b_{0}\right) \in f\right\} . B_{1}$ is finite; for if $x, y \in B_{1}$, then $\left(x, b_{0}\right),\left(y, b_{0}\right)$ are both acceptable loops at some stage of $C$; hence $y \in \hat{g}(x)+\hat{h}(x)=$ a finite set. Now, $\alpha \cdot \beta$ is infinite; and, applying Lemma 
4.3, we have that, for some $x_{1} \in B_{1}$, the set

$$
\begin{array}{r}
B_{1}^{*}=\left\{y \in \alpha \cdot \beta \mid\left(y, x_{1}\right) \in f \vee(\exists k>0)\left(\exists v_{1}, \cdots, v_{k} \in \alpha \cdot \beta\right)\right. \\
\left.\left(\left(y, v_{1}\right),\left(v_{1}, v_{2}\right), \cdots,\left(v_{k-1}, v_{k}\right),\left(v_{k}, x_{1}\right) \in f\right)\right\}
\end{array}
$$

is infinite. Let $c_{1}$ be the smallest such $x_{1}$ in $B_{1}$. Note that $\left(c_{1}, c_{0}\right) \in f$. Again, $B_{2}=\left\{y \in \alpha \cdot \beta \mid\left(y, c_{1}\right) \in f\right\}$ is finite; we may evidently iterate this argument ad infinitum. Thus, there exists a sequence $c_{0}, c_{1}, c_{2}, \ldots$ of distinct elements of $\alpha \cdot \beta$ such that $\left(c_{0}, c_{0}\right) \in f$ and $\left(c_{n+1}, c_{n}\right) \in f$ for all $n$. (The axiom of choice is not needed $\left({ }^{3}\right)$.) I

By Proposition 7 of [1] and the footnote on p. 342 of [4], we see that a number set $\alpha$ has an infinite regressive subset iff there is an r.e. sequence $\left\{\omega_{\varphi(n)}\right\}$ of pairwise disjoint r.e. sets satisfying $\omega_{\varphi(n)} \cdot \alpha \neq \emptyset$ for every $n$; moreover, if $\alpha$ itself is regressive, then there is such a sequence $\left\{\omega_{\varphi(n)}\right\}$, relative to $\alpha$, with the additional property that $\alpha \subseteq \bigcup_{n} \omega_{\varphi(n)}$. Hence, we might be led to formulate the following assertion, to which Theorem 4 would be a corollary:

Let $\alpha, \beta$ be number sets; and let $\left\{\omega_{\varphi(n)}\right\},\left\{\omega_{\psi(n)}\right\}$ be a pair of r.e. sequences of disjoint r.e. sets such that for every $n, \omega_{\varphi(n)} \cdot \alpha \neq \emptyset$ and $\omega_{\psi(n)} \cdot \beta \neq \emptyset$, and such that $\alpha \subseteq \bigcup_{n} \omega_{\varphi(n)}, \beta \subseteq \bigcup_{n} \omega_{\psi(n)}$. Then, if $\alpha \cdot \beta$ is infinite, there exists an r.e. sequence $\left\{\omega_{\zeta(n)}\right\}$ of disjoint r.e. sets such that $\omega_{\zeta(n)} \cdot \alpha \cdot \beta \neq \emptyset$ for every $n$.

But it is easily seen that this assertion is false: using the obvious fact that any infinite r.e. set is the union of a recursive set and a nonsimple r.e. set, we write $\gamma^{\prime}=\alpha \cdot \beta$, where $\gamma$ is hyperhypersimple, $\alpha^{\prime}$ is recursive, and $\beta^{\prime}$ is a nonsimple r.e. set; Theorem 6 of [4] shows that $\alpha, \beta$ provide a counterexample to the proposal in question.

CoROLlaRY 4.4. Let $\alpha$ be hyperhypersimple, $\beta$ hypersimple, $\beta^{\prime}$ regressive. Then $\alpha$ and $\beta$ are many-one incomparable.

Proof. (i) Suppose $\alpha \leqq_{m} \beta$ via $f$. Now $f\left(\alpha^{\prime}\right)$ must be an infinite subset of $\beta^{\prime}$, lest $\beta$ be recursive; hence, $f\left(\alpha^{\prime}\right)=\rho f \cdot \beta^{\prime}$ has an infinite regressive subset $\tau$, by Theorem 4. Define a partial recursive function $f$, with domain $\rho f$, as follows:

$$
\check{f}(x)=\left\{\begin{array}{l}
(\mu y)(f(y)=x), \quad \text { if } x \in \rho f ; \\
\text { undefined otherwise. }
\end{array}\right.
$$

Clearly $\check{f}$ is $1-1$; hence, by $[1$, Proposition $4(\mathrm{~b})], f(\tau)$ is an infinite regressive subset of $\alpha^{\prime}$. But this, in view of [1, Proposition 7] and [4, Theorem $6]$, is impossible. Hence, $\alpha \underset{m}{ } \beta$.

(3) The construction given in the proof of Theorem 4 exhibits the fact that the intersection of two retraceable sets is itself retraceable. (This observation is due to the referee; the fact in question is, presumably, well known.) Also, in connection with Theorem 4, we wish to thank Joseph Barback for pointing out to one of us, in a letter of January 27, 1964, an alternative line of proof for this theorem. 
(ii) Suppose $\beta \leqq_{m} \alpha$ via $g$. Define $\breve{g}$, relative to $g$, just as $f$ was defined relative to $f$ in (i). Then both $\check{g}$ and $\left.g\right|_{\rho g}$ are partial isomorphisms. Since $g\left(\beta^{\prime}\right)$ must be infinite, $\breve{g} g\left(\beta^{\prime}\right)$ is an infinite subset of $\beta^{\prime}$. Hence, by Theorem 4 , ğg $\left(\beta^{\prime}\right)$ has an infinite regressive subset $\lambda$; it follows that $g(\lambda)$ is an infinite regressive subset of $\alpha^{\prime}$, and so, as before, we have a contradiction. Thus $\beta$ 本 ${ }_{m} \alpha$. I

Corollary 4.4 is in contrast to the following result, established in [2]:

Proposition 4.5. If $\beta$ is hyperhypersimple, then there exists a hypersimple, nonhyperhypersimple set $\alpha$ such that $\alpha \equiv_{m} \beta$. (Indeed, given an index of $\beta$, we can effectively find an index of such a set $\alpha$.)

It is easily seen from elementary isomorphism and intersection properties that any hyperhypersimple set is the union of two hypersimple, nonhyperhypersimple sets. Note, however

Corollary 4.6. A hyperhypersimple set cannot be the union of two coregressive hypersimple sets.

Proof. By Theorem 4, [1, Proposition 7], and the easier half of $[4$, Theorem 6].

As a concluding remark about intersections and regressiveness, we observe that the following may be proved (though we do not prove it here): There exist hypersimple sets $\alpha$ and $\beta$ such that $\alpha^{\prime}$ and $\beta^{\prime}$ are regressive, but $(\alpha \cdot \beta)^{\prime}=\alpha^{\prime}+\beta^{\prime}$ is not regressive.

Added in proof. Theorem 4 can be made to read: if $\alpha_{1}, \cdots, \alpha_{k}(k \geqq 2)$ are regressive and $\bigcap_{j=1} k_{\alpha_{j}}$ infinite, then $\bigcap_{j=1} k_{\alpha_{j}}$ has an infinite regressive subset; the only modification required in the proof is a more general definition of loop. Again, Theorem 1 may be made to read thus: the union of any two immune regressive sets is immune; here the argument in $\$ 4$ suffices, though it is not clear how (or indeed, whether it is possible) to modify the proof in $\$ 3$ in order to get the stronger result. In connection with Theorem 1, we remark that the techniques of this paper seem entirely inadequate for setting, in the negative, the question: can the natural numbers be obtained as the union of three immune regressive sets?

\section{REFERENCES}

1. J. C. E. Dekker, Infinite series of isols, Proc. Sympos. Pure Math. Vol. 5, pp. 77-96, Amer. Math. Soc., Providence, R. I., 1962.

2. T. G. McLaughlin, Strong reducibility on hypersimple sets, Notre Dame J. Formal Logic (to appear).

3. E. L. Post, Recursively enumerable sets of positive integers and their decision problems, Bull. Amer. Math. Soc. 50 (1944), 284-316.

4. C. E. M. Yates, Recursively enumerable sets and retracing functions, Z. Math. Logik Grundlagen Math. 8 (1962), 331-345.

UNIVERSITY OF ILLINOIS, URBANA, ILLINOIS 